\title{
De los \\ controles \\ disciplinarios \\ a los \\ controles \\ securitarios
}

Pedro Oliver Olmo

M. ${ }^{a}$ Carmen Cubero Izquierdo (coords.) 

De los controles disciplinarios a los controles securitarios. Actas del II Congreso Internacional sobre la Historia de la Prisión y las Instituciones Punitivas 



\title{
De los controles disciplinarios a los controles securitarios. Actas del II Congreso Internacional sobre la Historia de la Prisión y las Instituciones Punitivas
}

\author{
Pedro Oliver Olmo \\ Ma Carmen Cubero Izquierdo \\ (Coords.)
}

(ภdiciones de la Universidad

Cuenca, 2020 
(C) de los textos: sus autores

(C) de las imágenes: sus atores

(C) de la edición: Universidad de Castilla-La Mancha

Edita: Ediciones de la Universidad de Castilla-La Mancha con la colaboración del Grupo de Estudios de Historia de la Prisión y de las Instituciones Punitivas.

Colección JORNADAS Y CONGRESOS, n. ${ }^{\circ} 25$

El procedimiento de selección de originales se ajusta a los criterios específicos del campo 10 de la CNEAI para los sexenios de investigación, en el que se indica que la admisión de los trabajos publicados para las actas de congresos deben responder a criterios de calidad equiparables a los exigidos por las revistas científicas.

17 Esta editorial es miembro de la UNE, lo que garantiza la difusión y comercialización de sus puCo blicaciones a nivel nacional e internacional.

Foto de cubierta: Prisioneros republicanos arreglando una carretera San Pedro de Cardeña, Burgos. 1938, Delespro. Recuperado de Biblioteca Digital Hispánica http://bdh-rd.bne.es/viewer. vm?id=0000227116\&page=43, CC-BY-NC-SA

ISBN: 978-84-9044-398-9

DOI: http://doi.org/10.18239/jornadas_2020.25.00

Esta obra forma parte de un proyecto de investigación de ámbito estatal que financia el MINECO, con el título "Del control disciplinario al control securitario en la España contemporánea" (referencia HAR2016-75098-R).

Apoya económicamente la edición: Facultad de Relaciones Laborales y RRHH (Campus de Albacete).

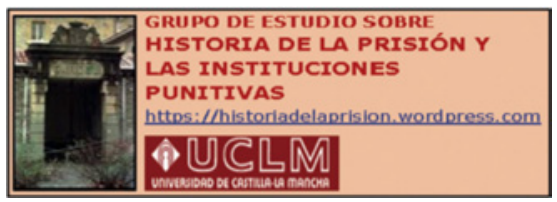

Composición: Compobell

Hecho en España (U.E.) - Made in Spain (E.U.)

\section{(C) $\oplus \Theta \Theta$}

Esta obra se encuentra bajo una licencia internacional Creative Commons BY-NC-ND 4.0. Cualquier forma de reproducción, distribución, comunicación pública o transformación de esta obra no incluida en la licencia Cretative Commons BY-NC-ND 4.0 solo puede ser realizada con la autorización expresa de los titulares, salvo excepción prevista por la ley. Puede Vd. acceder al texto completo de la licencia en este enlace: https://creativecommons.org/licenses/by-nc-nd/4.0/deed.es 


\section{Índice}

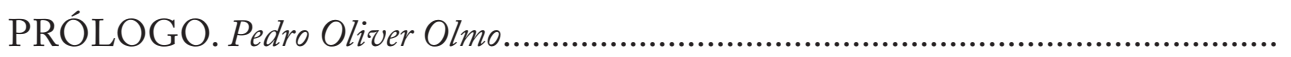

CONFERENCIA INAUGURAL. Un mar de luto. Represalias, control y represión de las mujeres en la dictadura franquista. Pura Sánchez...

PARTE I. INSTITUCIONES PUNITIVAS DURANTE EL ANTIGUO RÉGIMEN

Presentación. Pedro Oliver Olmo

Los graffiti carcelarios de la Edad Media y Moderna en la provincia de Ciudad Real: un estudio comparado. Victor Manuel López-Menchero Bendicho, Miguel Ángel Hervás Herrera, James Bart Mcleod, Jeffrey P. Du Vernay, Herbert D. G. Maschner, Manuel Retuerce Velasco, Honorio Javier Alvarez Garcia y Diego Lucendo......................

La Inquisición Española: Institución punitiva. Hayet Belhmaied................................. Ley, Orden y Castigo. El sistema punitivo de la Sala de Alcaldes de Casa y Corte en el Madrid del Antiguo Régimen. Francisco Javier Cubo Machado.

Las medidas represivas contra vagos y pobres como mecanismo de garantía del orden público en España durante la Edad Moderna. José Enrique Anguita Osuna y Álvaro Alzina Lozano.

E1 Hospicio como disciplina del pobre en la España Moderna: entre la "Misericordia" y la Penalidad". Victoria López Barahona y Alberto Morán Corte

La Cárcel Real de Sanlúcar de Barrameda: una carga insoportable para un cabildo municipal del Antiguo Régimen (1710-1820). Salvador Daza Palacios

To hold until satisfaction". Imprisonment for debt and carceral discipline in eighteenth century England. John Levin. 
PARTE II. PRISIÓN E INSTITUCIONES PUNITIVAS EN LOS ESTADOS LIBERALES

Presentación. Ángel Organero Merino y Pedro Oliver Olmo

Sistema penal no Liberalismo português: Discurso e práticas (1867-1913). Maria João Vaz.

Tiempo de aflicción: penas largas y penas muy largas en la prisión liberal. Luís Gargallo Vaamonde y Pedro Oliver Olmo.....

"Felicitaciones de la Sociedad de prisiones de París". El encierro indígena en Perú antes/después del Código penal de 1924. Jessica Ayre

La Colonia Penitenciaria en Ceuta (1889-1910) como tránsito del Sistema Disciplinario al Progresivo. La implicación de la burguesía del siglo XX. Antonio Carmona Portillo

El establecimiento penal de Ocaña: de prisión a reformatorio de adultos. Motivaciones para la reconversión y legislación aplicada. Ángel Organero Merino.

The minor detainees in the House of Supervised Education of the Prisons of Fresnes, 1929-1958. Jean-Lucien Sanchez.

PARTE III. PRISIÓN E INSTITUCIONES PUNITIVAS DURANTE EL FRANQUISMO

Presentación. Nuevas aportaciones de una historiografía aún demasiado cerrada. Fernando Mendiola Gonzalo y Juan Carlos Garcia-Funes.

Los Comités Madrileños como centros punitivos durante el otoño invierno de 1936. Fernando Jiménez Herrera.

$\mathrm{El}$ adoctrinamiento de los reclusos durante la Guerra Civil y Posguerra inicial. La Prisión Provincial de Córdoba. Francisco Navarro López

E1 Sistema Penitenciario Español en la posguerra. Un balance historiográfico. Juan Luis Castanedo Álvarez.

E1 Batallón disciplinario de trabajadores número 12,1940-1942. Un estudio de caso. Josep Màrius Climent.

Trabajos forzados en el franquismo o la pena como negocio. El caso de "Ponte Mourulle" (1942-1945). Prof. Dr. Uxio-Breogán Diéguez Cequiel y Prof. Dra. Sara Carou Garcia

Miranda de Ebro, campo de refugiados Aliados y del Eje: 1940-1947. Un enfoque transnacional. Concepción Pallarés Moraño

Memoria de un carabinero que perdió la vida en Güsen. Alicia Duñaiturria Laguarda

PARTE IV. LAS PRISIONES DE FINALES DEL SIGLO XX E INICIOS DEL XXI

Presentación. César Lorenzo Rubio, Eduardo Parra Iñesta, Débora Ávila Cantos, Sergio García García e Ignacio Mendiola Gonzalo

E1 Tratamiento Penitenciario como mecanismo de disciplina carcelaria. Hacia la superación del modelo autoritario de rehabilitación. Puerto Solar Calvo y Pedro Lacal Cuenca.

Transformaciones en las formas de ejercicio del poder penal en España en el siglo xxi: el caso de los Módulos de Respeto. Ana Ballesteros Pena

Sobre una pena infame: la Prisión Permanente Revisable. Y su extensión a aquellas de larga duración. Paz Francés Lecumberri. 
¿Tiene España un problema de sobrepoblación penitenciaria?. Cristina Rodríguez Yagüe

Consideraciones conceptuales en la comparación histórica de las tasas de encarcelamiento. Ignacio González Sánchez.

El personal funcionario de Instituciones Penitenciarias. Tramas de sentido en torno a la prisión. Denis Losada Varela.

La práctica de la tortura en España durante la Transición y los Gobiernos Democráticos: una visión de conjunto. Eduardo Parra Iñesta.

Securitización humanitaria: usos y abusos de la ayuda internacional al servicio del control y las prácticas securitarias. Alejandro Pozo Marín.

Mecanismos de Control Social y Tratamiento Punitivo en los programas socioeducativos de lucha contra la pobreza. Juan Ramón Rodriguez Fernández

Gubernamentalidad liberal, gestión securitaria y sistema punitivo. Mario Dominguez Sánchez-Pinilla y David J. Dominguez González.....

\section{PARTE V. GÉNERO Y PUNICIÓN}

Presentación. Ana Isabel Simón Alegre y Fernando Hernández Holgado

El Patronato de Protección a la Mujer: Centros de encierro y control moral para las mujeres caidas. Carmen Guillén Lorente....

Educadas y apartadas del vicio: el Patronato de Protección a la Mujer de Sevilla en los inicios del franquismo. Ana-Maria Montero-Pedrera.

Procesos contra la pornografía. La construcción del control sobre el erotismo en España: 1880-1936. Ma Carmen Cubero Izquierdo

Represión penal de las mujeres de Bizkaia: Prisión Provincial de Bilbao y Chalet Orue (1937-1942). Mónica Calvo Ortiz.

Malas entre las malas. Un análisis antropológico a las violencias, medicalizaciones y controles hacia las mujeres consumidoras de drogas entre rejas. Guadalupe Moreno Vicente.

Soldados que fueron presos, Cuba 1898: Arquetipo viril, ciudadanía y violencia. Ana I. Simón-Alegre.

PARTE VI. IDENTIDADES, POLÍTICA Y RESISTENCIA EN PRISIÓN.....

Comparezco con todo respeto en busca de justicia no de clemencia. Las cartas de quejas de los reclusos en las cárceles franquistas ante la Administración de justicia. $M^{a}$ Dolores Madrid Cruz.

E1 Ejercicio Peticionario de presos durante el Segundo Reinado Brasileño (Pernambuco/Rio Grande do Sul). Tiago da Silva Cesar....

Isolation, Control and Resistance: Political inmates in the Shlissel'burg fortress, 1884-1906. Dr Sarah J. Young

Repertorios de la acción colectiva en las cárceles de Colombia, 1990-2005. Miriam

Fajardo Gustin.

Dictadura y represión en Cuba: Violencia política y políticas de la violencia durante la Insurrección, 1952-1959. Manuel Ramírez Chicharro. 
Presas políticas y consolidación del franquismo en tiempos de postguerra: el caso de la Modelo de Barcelona. Carlota Sànchez Vidal.

Unimaginable Criminals: The disappearance of "Political Prisoners" in Spain and the West after 1945. Lucia Herrmann

Desplegarse para una acción eficaz de lucha contra la tortura en el mundo: la red SOS-Tortura de la OMCT (1985-2010). Pere Solà Gussinyer

\section{PARTE VII. CULTURAS Y PRÁCTICAS PUNITIVAS Y DE CONTROL EN} LA LARGA DURACIÓN

Presentación. Cristina de Pedro Álvarez y Daniel Oviedo Silva

Un acercamiento al estudio histórico de la Cárcel Municipal de Celaya como Institución de Control Social (1863-1961). Lic. Maria de los Angeles Arroyo Montoya.....

¿Está la Justicia Penal adaptada al menor? Un análisis histórico de la Justicia Juvenil. Esther Fernández Molina.

El doctor Ignacio Fernández Ortigosa y el establecimiento de los primeros Gabinetes de Antropometría Criminal en la cárcel de Belem (1894). Dr. Gerardo González Ascencio

Contra el espía enemigo. Los espacios de reclusión del Servicio de Investigación Militar Republicano durante la Guerra Civil española (1937-1939). Juan Carlos Marín Sánchez

La Reforma Penitenciaria Peronista en el extranjero: el asesoramiento de Roberto Pettinato en la construcción de la Penitenciaría del Litoral (Guayaquil, Ecuador, año 1954). Jorge A. Núñez.

Al otro lado de las rejas: la construcción del discurso periodístico sobre la prisión (1881-1923) . Victor José Ortega Muñoz. 


\title{
El tratamiento penitenciario como mecanismo de disciplina carcelaria. Hacia la superación del modelo autoritario de rehabilitación
}

\author{
Puerto Solar Calvo \\ Jurista II.PP. Doctora en Derecho \\ Pedro Lacal Cuenca \\ Psicólogo II.PP. Máster en Psicología Clínica \\ http://doi.org/10.18239/jornadas_2020.25.25
}

\begin{abstract}
RESUMEN
A pesar de que la lógica terapéutica indica que sólo un tratamiento voluntariamente aceptado puede tener éxito, la norma penitenciaria induce a cierta confusión sobre la naturaleza voluntaria u obligatoria del tratamiento penitenciario. Frente a la claridad con que se expresa el art. II2.3 $\mathrm{RP}$, al reconocer que "el interno podrá rechazar libremente o no colaborar en la realización de cualquier técnica de estudio de su personalidad, sin que ello tenga consecuencias disciplinarias, regimentales ni de regresión de grado", el art. 5.2 g) RP establece justamente lo contrario, catalogando como deber de los internos el de "participar en las actividades formativas, educativas y laborales definidas en función de sus carencias para la preparación de la vida en libertad". A su vez, la dinámica general de cumplimiento abona este poso normativo y la unión entre evolución tratamental y acceso a permisos, tercer grado y libertad condicional sirve más para promover la participación activa del interno en el tratamiento e, indirectamente, el respeto de la normativa regimental, que el verdadero cambio del mismo en cuanto a responsabilidad y autonomía personal.

Palabras clave: Tratamiento penitenciario, coacciones indirectas, modelo de rehabilitación autoritario, art. 25.2 CE.
\end{abstract}

\begin{abstract}
The logic of any rehabilitation treatment is that only voluntary accepted treatments can lead to good results. Nevertheless, some of the statements of the Spanish Penitentiary Rules introduce confusion in this field of study. According to art. II2.3 RP, inmates can regret any treatment without any kind of consequences. On the other hand, art. $5.2 \mathrm{~g}$ ) RP introduces the obligation of some of the activities that are usually considered as a part of the treatment. In this context, it is also important to consider that the link between treatment and access to penitentiary benefits as parole, is more useful for the prison as a way of pacification and indirect control of inmate's behavior, as for the inmate as a way to recover himself.
\end{abstract}

Keywords: Rison Treatment, indirect coercions, authority based rehabilitation model, art. $25.2 \mathrm{CE}$ 


\section{VOLUNTARIEDAD DEL TRATAMIENTO EN LA NORMA PENITENCIA- RIA}

Los arts. 7I LOPG y $73 \mathrm{RP}$ establecen como principio rector de la actividad penitenciaria, el sometimiento del régimen al tratamiento -"el fin primordial del régimen de los establecimientos de cumplimiento es lograr en los mismos el ambiente adecuado para el éxito del tratamiento"-, de manera que "las funciones regimentales deben ser consideradas como medios y no como finalidades en sí mismas" (art. 71.I LOGP). Sin embargo, la unión de ambos conceptos, régimen y tratamiento, y específicamente, el sometimiento del primero al segundo, tiene consecuencias ciertamente contradictorias y no deseables para lo que constituye el fin de la actividad penitenciaria -hacer de los internos ciudadanos responsables y autónomos capaces de vivir dentro de la legalidad-. De un lado, el concepto de tratamiento se difumina en el de régimen hasta el punto de considerar que casi cualquier actividad relacionada con lo penitenciario puede considerarse como tratamiento. Esto es, el mero cumplimiento de la normativa regimental se interpreta como evolución tratamental. De otro, al concepto de régimen se le asocian tintes terapéuticos que nada tienen que ver con su realidad y que acaban haciendo del régimen algo bueno y del tratamiento algo cuasi-obligatorio. Por mucho que unamos ambos conceptos, estos no dejan de ser distintos: el primero de carácter eminentemente normativo y de cumplimiento obligatorio para los internos ${ }^{\mathrm{I}}$, el segundo de naturaleza terapéutica, necesariamente voluntario.

Sin embargo, partiendo de esta unión conceptual entre régimen y tratamiento, el RP trata de forzar la participación del interno en la parte más tratamental de la ejecución de la condena. Si el cumplimiento de la normativa regimental es obligatorio, si el régimen en sí es terapéutico, tratamental y por ende, bueno para el interno, qué nos impide extender esa obligatoriedad al tratamiento si ambos conceptos están unidos. Los límites del fin de resocialización y de la centralidad del tratamiento se observan precisamente en la difícil salida a la disyuntiva régimen (disciplina) versus tratamiento (voluntariedad) (ZÚÑIGA RODRÍGUEZ, 200I: 3I8). De este modo se hace evidente una de las graves contradicciones internas de nuestra legislación penitenciaria, al convertir en columna vertebral del sistema de cumplimiento y en fin del régimen penitenciario un elemento del que el interno puede disponer (TAMARIT SUMALLA y GARCÍA ALBERO, 2005: 47).

Es la norma la que da muestra de esta contradicción cuando aborda el principio de voluntariedad del tratamiento penitenciario. A pesar de la claridad con que se expresa el art. II2.3 RP sobre el mismo, al reconocer que «el interno podrá rechazar libremente o no colaborar en la realización de cualquier técnica de estudio de su personalidad, sin que ello tenga consecuencias disciplinarias, regimentales ni de regresión de grado»², el art. $5.2 \mathrm{~g}$ ) RP establece justamente lo contrario, catalogando como deber de los internos el de «participar en las actividades formativas, educativas y laborales definidas en función de sus carencias para la preparación de la vida en libertad». Más significativos aún, el art. 26 LOGP que dispone que «el trabajo será considerado como un derecho y como un deber del interno, siendo un

1 El art. 4.1b) LOGP establece como deber de los internos: "Acatar las normas de régimen interior, reguladoras de la vida del establecimiento, cumpliendo las sanciones disciplinarias que le sean impuestas en el caso de infracción de aquellas, y de conformidad con lo establecido en el art. 44". De manera similar el art. 5.2b) RP determina el deber de: "Acatar las normas de régimen interior y las órdenes que reciba del personal penitenciario en el ejercicio legítimo de sus funciones".

2 En el mismo sentido y específicamente para los programas de tratamiento especializados, el art. 116.4 RP in fine determina que: "E1 seguimiento de estos programas será siempre voluntario y no podrá suponer la marginación de los internos afectados en los Centros Penitenciarios". Por su parte, el art. 117.5 RP para las medidas regimentales, establece que: "la participación en el programa podrá ser revocada por decisión voluntaria del interno, por el incumplimiento de las condiciones establecidas o por circunstancias sobrevenidas que justifiquen esta decisión”. 
elemento fundamental del tratamiento", y el art. $\mathrm{I}_{2} \mathrm{RP}$ que define el trabajo productivo en los siguientes términos: «(...) es un derecho y un deber del interno, constituye un elemento fundamental del tratamiento cuando resulte de la formulación de un programa individualizado, y tiene, además, la finalidad de preparar a los internos para el acceso al mercado laboral cuando alcancen la libertad» (CERVELLÓ DONDERIS, 20I6: 259). En la misma línea, los arts. 4.2 LOGP -dentro del catálogo de deberes de los internos- y 6r LOGP, inducen a la confusión al establecer que se fomentará la participación de los internos en el tratamiento y que éste «colaborará para, en el futuro, ser capaz de llevar, con conciencia social, una vida sin delitos» (MAPELLI CAFFARENA, 1983: 267). Lo anterior hasta el punto de que algunos autores sustentan la configuración de dicha participación como deber del condenado (ALARCÓN BRAVO, J., 1978: 30-31; GARRIDO GUZMÁN, I983: 295-296; BUENO ARÚS, 2006: 24-25).

No obstante, a pesar de la confusión que la norma genera, en la práctica, por la propia necesidad de concurrencia de la voluntad del interno para el éxito del tratamiento, se entiende que no cabe su desarrollo sin la misma (ARMENTA GONZÁLEZ-PALENZUELA y RODRÍGUEZ RAMÍREZ, 2006: 254; LEGANÉS GÓMEZ, 2005: 168-I69; VAN ZYL SMIT y SNACKEN, 2013: 300-30I). De ahí que la Administración sólo pueda, de acuerdo con el art. II2.I RP, estimular «la participación del interno en la planificación y ejecución de su tratamiento» 3 y que la no participación no pueda tener consecuencias negativas, tal y como señala el apartado 3 del art. II2 RP y desarrolla el art. II2.4 RP para el momento de la revisión de grado. Conforme a este último, «en los casos a que se refiere el apartado anterior, la clasificación inicial y las posteriores revisiones de la misma se realizarán mediante la observación directa del comportamiento y los informes pertinentes del personal penitenciario de los Equipos Técnicos que tenga relación con el interno, así como utilizando los datos documentales existentes».

Según este planteamiento dominante en la doctrina, el tratamiento constituye un derecho del interno que la Administración Penitenciaria ha de ofrecer y fomentar, pero nunca imponer, pues lo contrario convertiría la pretensión de cualquier logro terapéutico en inútil (FERNÁNDEZ ARÉVALO y NISTAL BURÓN, 2or6: 50I). Y todo ello, como decimos, sin que el rechazo del tratamiento surta consecuencias para el interno, tanto desde el punto de vista de una progresión de grado, como en relación al acceso a la dinámica de permisos o a cualesquiera beneficios penitenciarios (JUANATEY DORADO, 20I1: 152; GALLEGO DÍAZ, 2013: II4). Como fundamentos para esta postura, MAPELLI CAFFARENA (I983) aporta los siguientes:

En primer lugar, porque al tratado, al privarle de beneficios, no se le puede castigar más que al que no está necesitado de tratamiento. En segundo lugar, la ejecución de la pena del que no necesita tratamiento también está afectada por la resocialización de modo que no puede convertirse en una mera retención. En tercer lugar, porque la sentencia judicial entendida

$3 \mathrm{El}$ art. $55 \mathrm{RP}$ va más allá y regula que la Administración fomentará la participación de los internos en la propia organización de las actividades a realizar. De acuerdo con el mismo: "1. Los internos participarán en la organización del horario de las actividades de orden educativo, recreativo, religioso, laboral, cultural o deportivo. 2. También se procurará la participación de los internos en el desenvolvimiento de los servicios alimentarios y de confección de racionados, de acuerdo con el artículo 24 de la Ley Orgánica General Penitenciaria y de lo que se establezca en las normas de desarrollo de este Reglamento. 3. El Consejo de Dirección, mediante resolución motivada, podrá ampliar la participación de los internos en otras áreas regimentales diferentes de las mencionadas en el apartado 1 de este artículo. 4. La participación de los internos en estas actividades en los Establecimientos de cumplimiento de régimen abierto y de régimen ordinario y en los de preventivos, se efectuará a través de Comisiones ajustándose a las normas que desarrollan los siguientes artículos". Principio que el art. $79 \mathrm{RP}$ repite para el régimen ordinario y que ha dado lugar a los llamados Módulos de Respeto regulado en la Instrucción 18/2011, SG.II.PP., sobre Niveles de intervención en módulos de respeto. Esto es, módulos en los que se alcanza un alto nivel de autogestión regimental. 
como sanción abarca tanto a los reclusos que rechazan el tratamiento como a aquellos que lo aceptan. Y, en cuarto lugar, porque el fomento del consentimiento no dejaría de ser una falacia encargada de encubrir el tratamiento impuesto (p. 268).

\section{LA REALIDAD DEL TRATAMIENTO EN LA PRÁCTICA}

Sin embargo y a pesar de lo expuesto, la realidad práctica es otra bien distinta. Habitualmente, la no participación del interno en su tratamiento supondrá la falta de acceso a mayores cotas de libertad (ARMENTA GONZÁLEZ-PALENZUELA y RODRÍGUEZ RAMÍREZ, 2006: 254-255; GALLEGO DÍAZ, 20I3: IO5; CERVELLÓ DONDERIS, 2016: 242) En el mejor de los casos, los Equipos Técnicos no contarán con información suficiente para fundamentar dicho acceso a un régimen de cumplimiento más abierto. En el peor, la negativa del interno a participar en el tratamiento propuesto será entendida como negativa al cambio. En definitiva, en la práctica de las Juntas de Tratamiento no es infrecuente la invocación de la variable de "no participa en actividades de tratamiento", como desvalor de cara al inicio de la trayectoria de reinserción (RACIONERO CARMONA, r999: 24; RÍOS MARTÍN, J., 2005: 488-489).

De este modo, la dinámica general de cumplimiento abona el poso normativo introducido por los arts.5.2g) y I32 RP y la propia unión entre evolución tratamental y acceso a permisos, tercer grado y libertad condicional sirve más para promover la participación activa del interno en el tratamiento e, indirectamente, el respeto de la normativa regimental, que el verdadero cambio del mismo en cuanto a responsabilidad y autonomía personal. CERVELLÓ DONDERIS (20I6) resume la situación en los siguientes términos:

Su rechazo no puede provocar la imposición de sanciones, ni la regresión de grado, ni el uso de medios coercitivos, sin embargo el hecho de que su aceptación y colaboración activa sí tenga efectos positivos como el acceso a los beneficios penitenciarios, puede hacer pensar que no es tan voluntario como la propia legislación expresa (p. 242).

Aspecto por el que GALLEGO DÍAZ (2013: IO5) habla de las "coacciones indirectas" que pueden suponer la pérdida o el disfrute por parte del interno de determinas ventajas o beneficios si se deciden a optar por rechazar el tratamiento.

Así, paradójicamente y en contra de sus expectativas, el sistema favorece que la adaptación normativa del interno y su participación en el tratamiento sean "metas transitorias de alta rentabilidad" en detrimento de su auténtico cambio (ARMENTA GONZÁLEZ-PALENZUELA y RODRÍGUEZ RAMÍREZ, 2006: 255). El tratamiento queda reducido a mero instrumento a través del que acceder lo antes posible a la dinámica de permisos y el tercer grado. Como advierte LACAL CUENCA (2015):

Con este proceder hacemos saber a los internos que si quieren obtener algún beneficio, evitando el castigo que obtendrían en caso contrario, deben aceptar y cumplir con su tratamiento. Estamos forzando conductas sin ocuparnos de las emociones negativas, por la obligación de la que derivan, que a través de nuestra actuación generamos (...) En definitiva, no estamos cambiando actitudes. Estamos creando un sistema de recompensas (...) Las cosas que hacemos las hacemos no por lo que representan de mejora personal sino por lo que conllevan de beneficio a más o menos plazo, luego lo que hago lo hago por lo que obtengo circunstancialmente y no por lo que de mejora personal supone (p. Io).

Por esta vía, desde un punto de vista práctico y utilitario, se acaba premiando al interno más "prisionizado", al que asume la cultura carcelaria (GALLEGO DÍAZ, 20I3: II2). Esto es, tendrán más éxito, en cuanto al acceso a mayores cotas de libertad, aquellos que mejor partici- 
pen en la dinámica de cumplimiento que se les impone pero no necesariamente quienes hayan experimentado los cambios tratamentales necesarios (DAUNIS RODRÍGUEZ, 2016: 276). Con todo ello, se conforma una especie de teatro bien articulado en el que, aparentemente, los números y las dinámicas funcionan, pero donde nada es tan sencillo ni tan bueno como a priori aparenta.

\section{CONSECUENCIAS DE LA OBLIGATORIEDAD DEL TRATAMIENTO}

Como no podía ser de otro modo, la situación descrita produce consecuencias altamente relevantes tanto desde el punto de vista de la recuperación social de los condenados, como considerando sus derechos. En primer lugar, desde el punto de vista terapéutico, el sistema de recompensas en que se educa a los internos no funciona en el exterior de los centros y dificulta enormemente su adaptación posterior una vez acceden al mundo real. El medio social normalizado es más complejo y pocas veces contempla dinámicas de comportamiento tan simplistas. "Las mejoras personales se llevan a cabo por lo que significan para el individuo" (LACAL CUENCA, 2015: Io) y no por el beneficio que a corto o medio plazo esperamos obtener. A su vez, esta sutil obligatoriedad que se impone sobre los internos conlleva una infantilización de los mismos, atenta contra las bases de su propia dignidad ${ }^{4} \mathrm{y}$, de nuevo, limita su desarrollo como ciudadanos autónomos y responsables de sus actos. Como reconoce MAPELLI CAFFARENA (1989):

Esta identificación (entre régimen y tratamiento) que después vuelve a corroborarse a través de algunos preceptos de la legislación introduce un factor de excesiva subjetivización en el marco de la ejecución. La ley debe evitar y no potenciar que el interno se someta a éste con el fin de no perder una serie de beneficios penitenciarios ya que como se ha demostrado con abundante referencia empírica cuando el penado sólo se ve impulsado a colaborar con el fin de mejorar su condición penitenciaria la labor resocializadora generalmente termina fracasando (p. Ioo).

De hecho, el mismo autor, en un trabajo posterior, señala que el sistema tiene el grave inconveniente de que el interno procura simular una progresión para alcanzar un status penitenciario más beneficioso, tildando el resultado de "fraude terapéutico que termina convirtiéndose en un obstáculo para la propia labor de tratamiento" (MAPELLI CAFFARENA, 20II: 186).

En segundo lugar, si el régimen se subordina al tratamiento, si, el cumplimiento de la normativa regimental y la realización de actividades pautadas se consideran tratamiento en sí, la conclusión es que el régimen como tal es algo bueno para el interno, con alto nivel terapéutico y escaso rigor normativo. Esta premisa absolutamente idealizada de lo que conlleva la estancia en prisión (TAMARIT SUMALLA y GARCÍA ALBERO, 2005: 259), resulta en la imposición de cierto buenismo penitenciario según el cual lo que la institución demanda al interno es por definición bueno para él. Ello tanto en el aspecto más tratamental, justificando la obligatoriedad que venimos analizando, como en el más regimental, provocando una rebaja importante de los derechos y garantías de quienes se encuentran en prisión. De acuerdo con RIVERA BEIRAS (2009):

Es necesario destacar que la configuración de la pena (privativa de libertad) como tratamiento, ha ido provocando paulatinamente que los derechos fundamentales de los reclusos condenados se conviertan en meros "beneficios penitenciarios", que se concederán o denegarán en

4 Ello en contra del art. 23 de la Instrucción 2/2011, SG.II.PP., sobre el Código Deontológico, SG.II.PP., que impone el respeto a la dignidad en los siguientes términos: "El personal penitenciario evitará el uso de formas o métodos de cumplimiento de la función pública que atenten contra la dignidad o la propia estima de las personas sometidas a sanciones o medidas penales, absteniéndose en especial de emplear términos o gestos devaluadores, discriminatorios o denigrantes". 
función de una constante evaluación de la conducta y de la personalidad de aquellos. Así, las posibilidades de disfrutar de salidas temporales o de ver anticipada incluso su libertad, se reducirán en función de aquellos criterios óptimos; eso sí, para lograr el gobierno disciplinario de la cárcel. La devaluación de los derechos fundamentales de los presos, con la consiguiente configuración de un "ciudadano de segunda categoría" se va perfilando claramente con esta tecnología punitivo-premial (p. 19).

\section{PROPUESTAS DE CAMBIO}

En este contexto, parte de la doctrina refiere que sólo una desvinculación absoluta entre beneficios penitenciarios y actividades de reinserción o tratamiento permite la voluntariedad de éste y rompe la dinámica que exponemos (MANZANARES SAMANIEGO, I984: 46). Siendo esto cierto, consideramos sin embargo que la solución es posible sin romper con la estructura que dibuja nuestro actual sistema penitenciario. Como advierten otros autores, se trata de escoger entre entender los beneficios en coherencia con la declaración del art. 25.2 CE (...), o calificarlos como medidas realistas adoptadas por simples razones de utilidad práctica para el mejor funcionamiento del aparato penitenciario (GARCÍA ARÁN, I983: IIO-II2 y II9).

La situación descrita asume el modelo autoritario de rehabilitación definido por ROTMAN (I994). De acuerdo con el mismo, y en contraposición a lo que el autor denomina modelo de rehabilitación antropocéntrico, enfocado al cliente y de carácter básicamente voluntario:

El modelo autoritario de rehabilitación, en realidad, no es otra cosa que una versión sutil del antiguo modelo represivo, el cual buscaba el cumplimiento de la misma a través de la intimidación y la coerción. La rehabilitación, en este contexto, es esencialmente un dispositivo técnico que está orientado a moldear al delincuente y asegurar que se adecúe a un patrón predeterminado de pensamiento y comportamiento (p. 292).

Del mismo modo, adaptado a nuestro contexto normativo, CUTIÑO RAYA (2015) define el tratamiento, más que como unos medios para alcanzar la resocialización de la persona penada, de la siguiente manera:

Como una táctica de disciplinamiento. Si la persona acata las normas de régimen interno, si su conducta es adecuada, podrá participar en las actividades educativas, recreativas o culturales, tendrá unas condiciones de vida mejores, disfrutará de permisos. Si es una persona conflictiva, inadaptada o incorregible, sus condiciones empeorarán y sus derechos serán más restringidos (p. 35).

De ahí que MCNEILL (20I7) destaque la importancia de que en todo proceso rehabilitador la persona «debe ser tratada como un sujeto moral y como otro ciudadano más, en vez de ser considerado como un mero individuo portador de riesgo, el cual debe ser manipulado o ajustado según los intereses de los demás" (p. 8).

Para dar el salto que reclamamos desde un modelo de rehabilitación autoritario a otro antropocéntrico, de corte radicalmente humano, es necesario modificar a su vez aspectos estructurales de nuestro sistema de ejecución que contribuyen al mantenimiento de este status quo que describimos. Así en primer lugar, sería necesario acabar con el tan denostado concepto de relación de sujeción especial que sigue justificando la merma generalizada de derechos jurídicos de los internos en prisión. En segundo lugar, y con tal finalidad, la relación de los internos con la Administración Penitenciaria habría de encuadrarse en los términos que de manera concreta señala el art. 25.2 CE. De un lado, con carácter general, la limitación de los derechos de los internos sólo es posible de forma restrictiva si específicamente lo exige el cumplimiento 
de la privación de libertad de modo justificado. De otro lado, en contra de lo que ahora viene sucediendo, habría que reconocer la reinserción y el tratamiento como auténticos derechos subjetivos susceptibles de amparo. Como acertadamente señala TÉLLEZ AGUILERA (I999):

El desafortunado eslogan penitenciario de «odia el delito y compadece al delincuente», desde el frontispicio arqueado de las viejas prisiones españolas, ha ejemplificado una filosofía de doble moral en la que el delincuente mientras está en la calle cometiendo delitos es un ser desalmado que las Fuerzas de Seguridad deben de reprimir sin tregua, pero cuando traspasa el umbral de la prisi6n, el delito queda en la calle y el hombre entra en el «mundo de la reinserción», donde todos lo tratarán para lograr que vuelva felizmente a la libertad y pueda llevar una vida sin delitos. Idealiza la idea que sóro pueden creer las mentes más ingenuas y que, en el fondo, convierte al interno en un ser minusválido y necesitado de ayuda; una pobre criatura de Dios sobre la que el Estado debe intervenir misericordemente (...) Por eso, a diferencia de la acogida de la idea de tratamiento, la de que el delincuente es un ciudadano que no se encuentra excluido de la sociedad, un sujeto activo dotado de derechos que puede ejercitar ante la Administraci6n y los Tribunales, sí que ya no fue acogida en nuestro país con tanto agrado. Por ello, se hace patente la necesidad de partir de una concepción del delincuente ajena a la doble moral antes criticada. La apuesta porque el interno es un ciudadano como otro cualquiera sujeto a la Administración por una relación jurídica cuyo marco jurídico viene encabezado por el art. 25.2 del texto constitucional e integrado por una serie de derechos que no pueden ser limitados por una vetusta teoría decimonónica, permitirá desterrar esa tan arraigada como errónea creencia de que el recluso es un ser inerte, sometido a la Administración, la cual, siguiendo un «mandato del legislador» dirige su actuación desde parámetros reinsertadores. Reconocer la existencia de un auténtico derecho a la reinserción social del delincuente, sin miedos infundados ni ambages, se torna una asignatura pendiente, que una vez superada permitiría concebirlo como un sujeto activo que exige la realizaci6n de sus derechos y no un mero sujeto pasivo de la bondad y misericordia de la Administración (pp. 326 y 334).

Y es que, en realidad, el estatus jurídico de los internos en nuestro sistema penitenciario no es tan beneficioso como pudiera inferirse de un primer análisis del mismo. Primar al tratamiento antes que al régimen conforme a los arts. 7I LOGP, $73 \mathrm{RP}$ puede ser bueno a los fines de generación del mejor ambiente de cumplimiento. Sin embargo, configurada bajo este prisma, la pena privativa de libertad adquiere un toque demasiado optimista, olvidando que ante todo, la pena en sí es un mal. Olvido que sin duda contribuye a que nuestro ordenamiento penitenciario se resista a dar carpetazo a conceptos como el de relación de sujeción especial, que han contribuido a que el poder de la Administración se manifieste en ocasiones omnipotente.

\section{EXCURSO. TRATAMIENTO PENITENCIARIO Y PPR}

Finalmente, cerramos esta reflexión con una cuestión no menos relevante a las ya apuntadas y que aporta actualidad a la temática seleccionada, pues la revisión de la prisión permanente revisable y el cese del internamiento que supone dependen entre otros, de la satisfactoria realización del tratamiento.

En términos generales, la introducción en nuestro ordenamiento de la prisión permanente revisable es la que más claramente responde, de entre las modificaciones normativas producidas en los últimos años, a los postulados del populismo punitivo (CERVELLÓ DONDERIS, 2016: II9). A través de la misma se trata de castigar con la mayor dureza a los considerados máximos enemigos de la seguridad ciudadana. Se consolida así la tendencia a configurar un Derecho Penal sobre la base de postulados imposibles, guiado por el criterio de la tolerancia cero, pero de alto contenido simbólico fácilmente asumible por la sociedad. A su vez, una vez metidos en la vorágine de endurecimiento, reconducir la norma a parámetros más racionales se torna más 
difícil. Si lo que se pretende es una especie de seguridad absoluta y dado que ésta por definición es inalcanzable, siempre habrá justificación para que la norma penal aumente en dureza y para que esos enemigos de ésta sean cada vez más numerosos.

En paralelo, la libertad condicional se ha convertido en medida alternativa a lo que venía siendo hasta el momento. Es decir, de ser el cuarto grado de cumplimiento de la pena privativa de libertad, ha pasado a ser una manera de suspender su ejecución. Aspecto que no sólo sirve para endurecer su régimen con carácter general a través de los apartados 5 y 6 del art. $90 \mathrm{CP}$, sino para permitir la revisión de la prisión permanente revisable en los durísimos términos del art. $92 \mathrm{CP}$, dotando a esta pena de una apariencia de legitimidad también en el plano constitucional, al permitir que sea una pena temporalmente determinable.

Tanto la introducción de la pena de prisión permanente revisable, como su específica configuración jurídica, han sido duramente criticadas. En este sentido, son muchas las manifestaciones de la doctrina contrarias a la prisión permanente revisable desde el punto de vista de su incompatibilidad con diferentes preceptos de nuestra CE. En especial, los arts. I5 y 25.2 CE (ARROYO ZAPATERO et al. 2016). Sin embargo, pocos son los estudios que ha relacionado la prisión permanente revisable con el tratamiento penitenciario y, más estrechamente, con el carácter necesariamente voluntario del mismo.

Para establecer esta relación, volvemos al principio, recordando el acuerdo doctrinal mayoritario sobre la necesaria voluntariedad del tratamiento para que éste sea eficaz desde un punto de vista terapéutico. Recordando lo dicho, la doctrina mayoritaria asegura que el tratamiento penitenciario ha de ser voluntario en el sentido que recoge el art. In2 RP. Sin embargo, la revisión de la prisión permanente revisable y el cese del internamiento que supone dependen entre otros, de la satisfactoria realización de dicho tratamiento. Con ello, se dan varias consecuencias altamente cuestionables desde el punto de vista de las garantías jurídicas. Primero, que se acepta por la norma penal que la privación de libertad sea perpetua para quien, ejercitando su derecho a no realizar tratamiento, no acepte llevar a cabo el mismo. Segundo, consecuencia de lo anterior, que una garantía jurídica de primer orden, como es la certeza de la condena y la seguridad jurídica de la que deriva, se hace depender de la voluntad del sujeto al que esa garantía ampara.

Configuración bastante llamativa, no sólo por sí misma, sino porque para que pueda concurrir la garantía de la certeza del fin de la condena, se compele al interno para que renuncie a otro derecho, el de no someterse a tratamiento alguno ¿Puede verdaderamente una garantía jurídica básica en nuestro ordenamiento, depender de la voluntad de quien se ha de beneficiar de dicha garantía? De nuevo, de este modo, ¿No estamos obligando a quien ha de ser per se beneficiario de la garantía de certeza del fin de su condena, a renunciar a su derecho a no llevar a cabo ningún tipo de tratamiento penitenciario, si efectivamente desea que la garantía referida pueda darse en la práctica?

\section{ALGUNAS CONCLUSIONES}

Como conclusiones básicas del trabajo desarrollado podemos alcanzar las siguientes:

La norma penitenciaria es confusa en la definición de la voluntariedad u obligatoriedad del tratamiento en prisión y así se deriva de la comparación entre el art. art. II2.3 RP y el art. 5.2g) RP.

A pesar de que la mayoría doctrinal no entiende que un tratamiento pueda ser exitoso sin la voluntad de quien es tratado, lo cierto es que la práctica penitenciaria provoca que el tratamiento tenga tintes claramente coactivos.

Las consecuencias de lo anterior son múltiples y afectan tanto a la auténtica recuperación social de los internos, como a su estatus jurídico y la satisfacción real de sus derechos. 
Desde el punto de vista terapéutico, se produce un fraude en virtud del cual los internos avanzan en el tratamiento en tanto obtienen los resultados cortoplacistas -en forma de beneficios penitenciarios- que desean.

Desde el punto de vista de los derechos de los internos, la permeabilidad conceptual entre régimen y tratamiento (arts.71 LOGP y $73 \mathrm{RP}$ ), provoca que se considere que el régimen como tal es algo bueno para el interno, con alto nivel terapéutico y escaso rigor normativo, provocando una rebaja importante de los derechos y garantías de quienes se encuentran en prisión.

El cambio a un modelo rehabilitador humanista y respetuoso con los derechos de quienes siguen siendo ciudadanos pasa por abordar una serie de cambios estructurales, pero que encuentran perfecto encaje en las bases normativas de nuestro sistema.

En primer lugar, sería necesario acabar con el tan denostado concepto de relación de sujeción especial que sigue justificando la merma generalizada de derechos jurídicos de los internos en prisión.

En segundo lugar, y con tal finalidad, la relación de los internos con la Administración Penitenciaria habría de encuadrarse en los términos que de manera concreta señala el art. 25.2 CE.

De un lado, con carácter general, la limitación de los derechos de los internos sólo sería posible de forma restrictiva si específicamente lo exige el cumplimiento de la privación de libertad de modo justificado.

De otro lado, en contra de lo que ahora viene sucediendo, habría que reconocer la reinserción y el tratamiento como auténticos derechos subjetivos susceptibles de amparo.

Por último, la actual regulación de la PPR nos sirve como claro ejemplo de la confusión de la naturaleza jurídica del tratamiento penitenciario. El carácter eminentemente voluntario de éste último vuelve a ser puesto en entredicho, al hacer depender una garantía jurídica básica -la determinación final de la condena- del ejercicio de un derecho del interno.

\section{BIBLIOGRAFÍA}

ALARCÓN BRAVO, J. (1978). “El tratamiento penitenciario”, en $E P y C$, n. 2, pp. I3-42.

ARMENTA GONZÁLEZ-PALENZUELA, F. J. y RODRÍGUEZ RAMÍREZ, V. (2006). Reglamento penitenciario comentado: análisis sistemático y recopilación de legislación. Madrid. MAD.

ARROYO ZAPATERO, L. A., LASCUARAÍN SÁNCHEZ, J. A., PÉREZ MANZANO, M.

(Coord.) (20I6). Contra la cadena perpetua. Universidad Castilla-La Mancha.

BUENO ARÚS, F. (2006). "Notas sobre la Ley General Penitenciaria” en REP, n. 252, pp. 9-36.

CERVELLÓ DONDERIS, V. (2016). Derecho Penitenciario. Valencia. Tirant lo Blanch.

CUTIÑO RAYA, S. (2015). "Algunos datos sobre la realidad del tratamiento en las prisiones españolas" en $R E C P C$, n. I7-II, pp. I-4I.

DAUNIS RODRÍGUEZ, A. (2016). "Criterios para la valoración de la peligrosidad y el riesgo en el ámbito penitenciario" en $C P C$, n. I20, pp. 239-280.

FERNÁNDEZ ARÉVALO, L., NISTAL BURÓN,J. (20I6). Derecho Penitenciario. Navarra. AranzadiThomson Reuters.

GALLEGO DÍAZ, M. (20I3). “Tratamiento penitenciario y voluntariedad" en REP, n. extra, in memoriam Profesor Francisco Bueno Arús, pp. Ioo-IIg.

GARCÍA ARÁN, M. (1983). “Los nuevos beneficios penitenciarios: una reforma inadvertida” en Revista Jurídica de Cataluña, n. I, pp. Io9-I24.

GARRIDO GUZMÁN, L. (1983). Manual de Ciencia Penitenciaria. Madrid. Edersa. 
JUANATEY DORADO, C. (2oII). Manual de Derecho penitenciario. Madrid. Iustel.

LACAL CUENCA, P. y SOLAR CALVO, P. (20I5). "Responsabilidad penal y personal. La incidencia de la LO I/2015 en el trabajo penitenciario" en Diario La Ley, n. 8591.

LEGANÉS GÓMEZ, S. (2005). Evolución de la clasificación penitenciaria. Madrid. Premio Nacional Victoria Kent, 2004. Ministerio del Interior.

MANZANARES SAMANIEGO, L. (1984). Individualización cientifica y libertad condicional. Madrid. Centro de Publicaciones del Ministerio de Justicia.

MAPELLI CAFFARENA, B. (20II). Las consecuencias jurídicas del delito. Pamplona. Aranzadi.

MAPELLI CAFFARENA, B. (1989). "La crisis de nuestro modelo legal de tratamiento penitenciario" en Eguzkilore, n. extra 2, pp. 99-II2.

MAPPELI CAFFARENA, B. (1983). Principios fundamentales del sistema penitenciario español. Barcelona. Bosch.

MCNEILL, F. (20I7). "Las consecuencias colaterales del riesgo" en InDret, n. I.

RACIONERO CARMONA, F. (1999). Derecho penitenciario y privación de libertad. Una perspectiva judicial. Madrid. Dykinson.

RÍOS MARTÍN, J. (2005). En Bueno Arús, F. Ley General Penitenciaria. Comentarios, jurisprudencia, doctrina. Madrid. Colex.

RIVERA BEIRAS, I. (2009). La cuestión carcelaria. Historia, Epistemología, Derecho y Politica Penitenciaria. Buenos Aires. Editores del Puerto.

ROTMAN, E. (1994). "Beyond punishment" en Duff, A., Garland, E. A reader on punishment. Oxford University Press.

TAMARIT SUMALLA, J. M., GARCÍA ALBERO, R., et al. (2005). Curso de Derecho penitenciario. Valencia. Tirant lo Blanch.

TÉLLEZ AGUILERA, A. (1999). "Retos del siglo XXI para el sistema penitenciario español” en $A D P C P$, t. 52, pp. 323-338.

ZÚÑIGA RODRÍGUEZ, L. (200I). "El tratamiento penitenciario I" en Berdugo Gómez de la Torre, I. et al. Manual de Derecho Penitenciario, Madrid. Colex.

VAN ZYL SMIT, D., SNACKEN, S. (2013). Principios de Derecho y Politica Penitenciaria Europea. Valencia. Tirant lo Blanch. 